\title{
Putting MicroED to the Test: An Account of the Evaluation of 30 Di- verse Pharmaceutical Compounds
}

\author{
Jessica E. Burch ${ }^{1 *}$, Austin G. Smith ${ }^{2 \dagger}$, Seb Caille $^{2}$, Shawn D. Walker ${ }^{2 \dagger}$, Ryan Wurz ${ }^{3}$, Victor J. Cee ${ }^{3 \dagger}$, \\ Jose Rodriguez ${ }^{1}$, D. Gostovic ${ }^{4}$, Kyle Quasdorf ${ }^{2 *}$, Hosea M. Nelson ${ }^{1,5 *}$ \\ ${ }^{1}$ Department of Chemistry and Biochemistry, University of California, Los Angeles, Los Angeles, CA 90095, USA. \\ ${ }^{2}$ Pivotal and Commercial Synthetics, Drug Substance Technologies, Process Development, Amgen Inc., One Amgen Center \\ Drive, Thousand Oaks, California 91320, USA. \\ ${ }^{3}$ Medicinal Chemistry, Amgen Inc., One Amgen Center Drive, Thousand Oaks, California 91320, USA. \\ ${ }^{4}$ MicroEDLab.com, 1623 Central Ave. Ste. 18, Cheyenne, Wyoming 82001, USA. \\ ${ }^{5}$ Division of Chemistry and Chemical Engineering, California Institute of Technology, Pasadena, California 91125, USA.
}

*Correspondence to: jess@microedlab.com, quasdorf@amgen.com, hosea@chem.ucla.edu

Supporting Information Placeholder

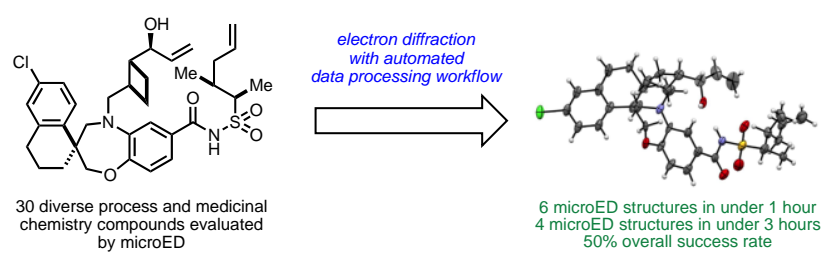

ABSTRACT: The application of microcrystal electron diffraction (microED) to a variety of pharmaceutical compounds is reported.
The examples and work detailed showcase the utility of microED as a routine technique for the rapid collection, analysis, and gener-
ation of structural data on a number of pharmaceutically relevant compounds, requiring minimal sample preparation and often without
the need for time-consuming vitrification and cryo transfer processes. The development of a scripted data processing workflow al-
lowed for simultaneous collection and processing of electron diffraction data, further expediting structural analysis of fifteen com-
pounds.

\section{Introduction.}

From process to medicinal chemistry, structural determination of key intermediates and final drug compounds are critical to establishing process understanding and protecting intellectual property. ${ }^{1}$ As the chemical complexity of the pipeline of compounds in the pharmaceutical industry increases (Figure 1), routine spectroscopic techniques such as NMR become more time consuming and may fail to provide unambiguous structural determination. ${ }^{2}$ The development of novel analytical techniques capable of rapidly obtaining unambiguous structural information from these complex species is crucial for the future of drug development.
Single crystal crystallography provides the most detailed structural information from the solid state, but traditionally employed X-ray crystallography is limited in practice by stringent crystal quality and size requirements. ${ }^{3}$ For samples that fail to produce crystals of sufficient quality for single crystal X-ray analysis, X-ray powder diffraction and NMR crystallography have been developed as alternative analytical techniques. These techniques may remain ineffective at providing unambiguous assignments in the case of highly complex compounds and also may fail to detect minor impurities. ${ }^{4-9}$ Information not only on structural elucidation, but also on variation in crystal forms is also key for reproducible and scalable processes. ${ }^{1}$ 


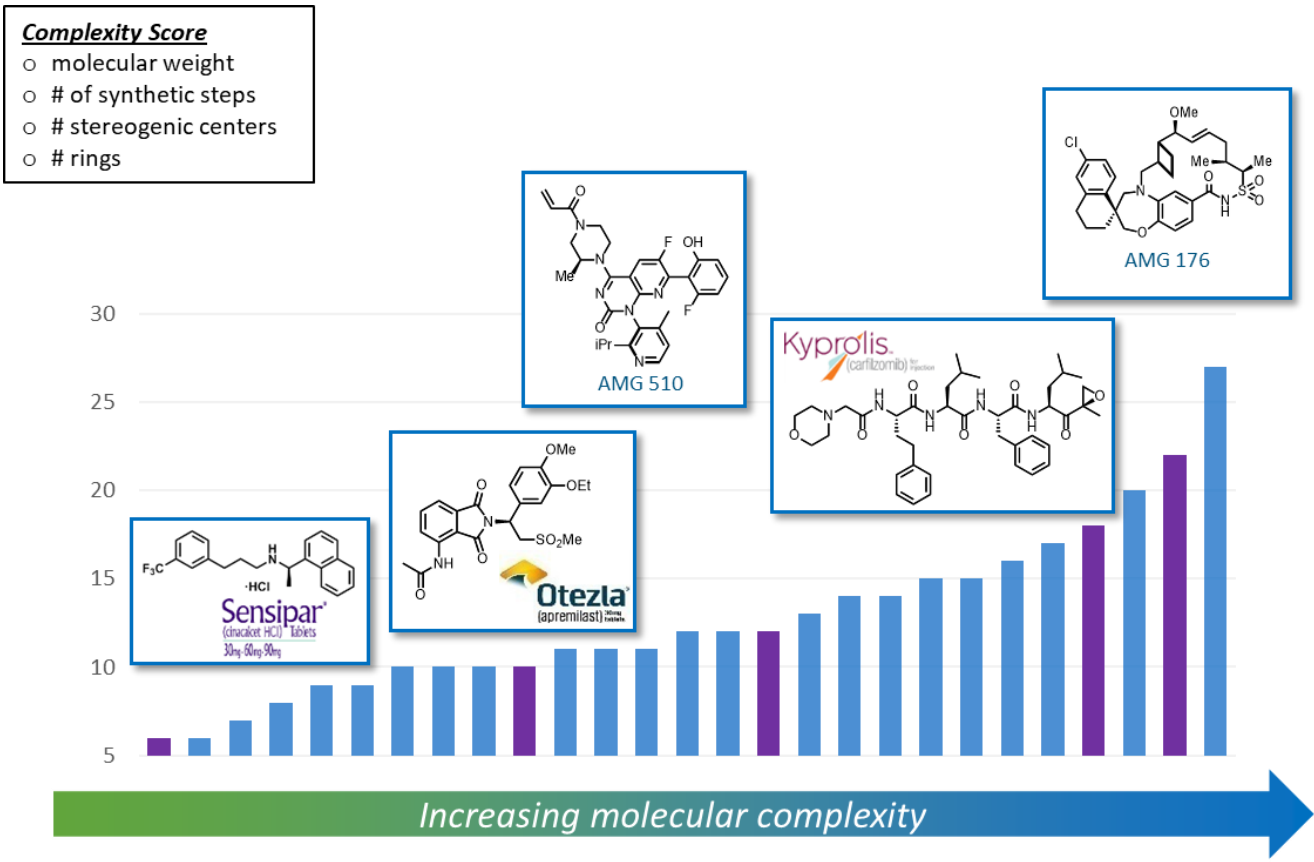

Figure 1. Molecular complexity score ranking the pipeline of Amgen's small molecule pharmaceutical programs.

Microcrystal electron diffraction (microED) has recently increased in popularity as a crystallographic technique capable of structural determination utilizing microcrystals that may be present in even seemingly amorphous powders. A number of reports have compared structural solutions of identical species obtained by both single crystal X-ray crystallography and microED, demonstrating the ability for microED to provide data equivalent in accuracy to the current gold standard in solid state structural assignment. ${ }^{10}$ Electron diffraction has been used to elucidate structures spanning a wide range of chemical space, from proteins, materials, and MOFs, to small organic molecules, complex natural products, and organometallic species. ${ }^{11-}$

${ }^{17}$ Furthermore, recent work determining the absolute configuration of a nanocrystalline pharmaceutical compound suggests a promising future in which microED can be utilized to determine absolute stereochemistry. ${ }^{18}$ In addition to its promise as a novel, routine crystallographic technique, microED is capable of detecting polymorphology and crystalline impurities on the nanomolar scale. ${ }^{19}$ This sensitivity is particularly attractive for industrial applications, where detection of minor impurities and crystal polymorphology could offer commercial value.

The purpose of this study was to evaluate the practicality of using microED as a tool to routinely determine chemical structures of pharmaceutically relevant compounds. Thirty pharmaceutical samples were selected for evaluation by microED, spanning a range of chemical complexity from both medicinal and process sectors. A subset of samples were isolated directly following routine chromatographic purification with no attempts to promote crystallinity, while another subset were isolated through purification by recrystallization.

\section{Materials and Methods.}

Materials and Initial Screening on the TEM. Initial efforts began by rapidly screening all thirty samples to evaluate crystallinity and collect diffraction datasets during the initial screening if possible. Samples were prepared by transferring milligram quantities of dry powder as received into a dram vial and manually grinding with a glass pipette. A pure carbon 200 mesh $\mathrm{Cu}$ grid was dropped into the vial and shaken together with the sample. The grid was removed and gently tapped while held with tweezers to remove excess powder. The sample was placed onto a single tilt holder and inserted into a Thermo Fisher Scientific Talos F200C transmission electron microscope equipped with a Ceta-D detector operating at an accelerating voltage of $200 \mathrm{keV}$.

During this initial screening period, active microscope time was limited to one hour or less per sample. To screen for crystallinity, particles were located on the grid in imaging mode at 2600x magnification (see Supporting Information Section 2 for images of this process). After identifying a particle of interest, a diffraction pattern was recorded by isolating a region of the particle using a selected area aperture and entering parallelilluminated diffraction mode utilizing the low dose software on the Thermo Fisher microscope user interface.

A single image of the diffraction pattern was taken on a Thermo Fisher Scientific Ceta-D camera. If user inspection of the diffraction pattern suggested that the particle was monocrystalline and provided $<1.2 \AA$ resolution diffraction, the eucentric height of the sample was finely adjusted in imaging mode to ensure the crystal would remain within the selected area aperture throughout a tilt series with a maximum tilt range of $\pm 65^{\circ}$.

After making these adjustments and returning to diffraction mode, a continuously rotating electron diffraction movie was collected by rotating the stage at a rate of $0.3^{\circ} \mathrm{s}^{-1}$. The Ceta-D CMOS $4 \mathrm{k}$ x $4 \mathrm{k}$ camera was operated using rolling shutter mode and continuously integrated at a rate of 3 seconds per frame with binning by 2 to produce $2 \mathrm{k}$ x $2 \mathrm{k}$ images. Diffraction movies were saved as SER files. 


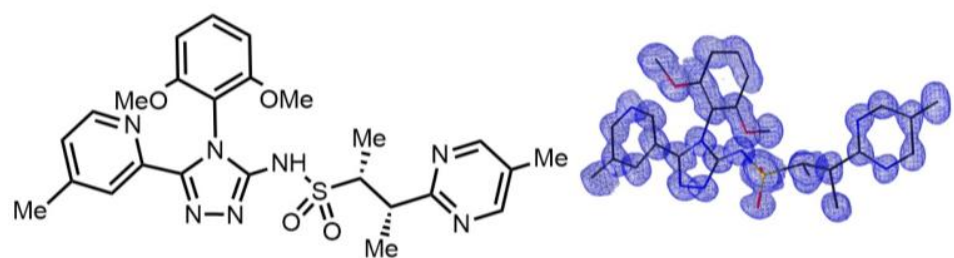

1

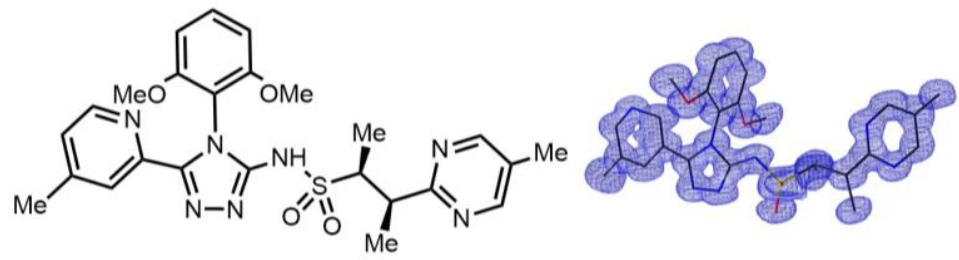

3

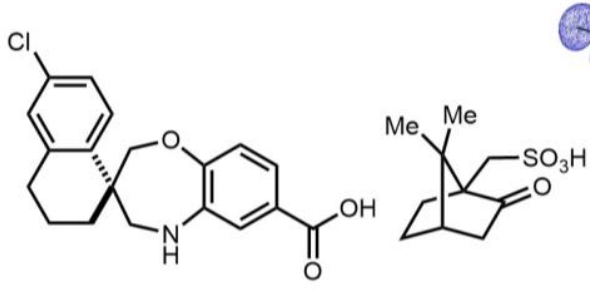

5
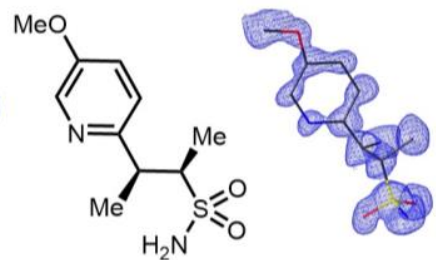

2

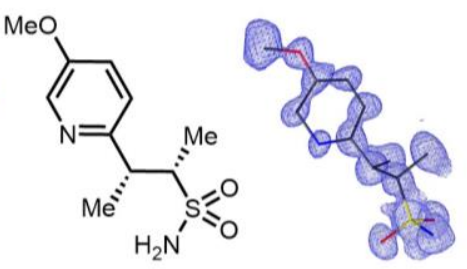

4
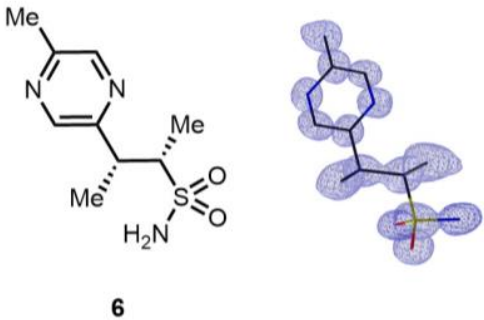

Figure 2. Structures in which a preliminary microED solution was obtained in under one hour each. Structures $\mathbf{1}$ and $\mathbf{3}$ have two molecules within their asymmetric unit (See Supporting Information). Hydrogens omitted for clarity. Structure $\mathbf{5}$ resolves the spirocyclic compound but fails to assign stereochemistry at the CSA due to disorder.

Streamlined Data Processing. Given the ability to collect multiple movies in a matter of minutes, to further expedite structural analysis, we wanted the ability for a single user to simultaneously collect and process data. To facilitate this, our laboratory has developed a Python script that interacts with existing programs utilized for processing microED data to allow for automated conversion and indexing, inspired by similar automation developed for serial rotation electron diffraction. ${ }^{20}$ Every movie collected for this study was successfully indexed and scaled using this strategy, taking approximately 1-2 minutes to process each dataset.

Movie files are saved with sample name, detector distance, stage rotation speed, and camera integration time separated by underscores, allowing for automated importing of these values into the processing software (See Supporting Information Sections 11-12). After executing the Python script, an open source movie conversion program is called to convert each SER movie to individual SMV frames. ${ }^{21}$ After conversion, the Python script writes a custom XDS.INP file with the appropriate settings based on our TEM settings and attempts to index and integrate frames using XDS. ${ }^{22}$ The script is capable of detecting errors commonly encountered from our previous processing efforts and automatically corrects and re-subjects the data to XDS processing as needed until the dataset is either successfully integrated, or an upper limit is reached and the data is determined to be unindexable by the automated program. Furthermore, the script generates and executes the scaling program XSCALE and converts the data to SHELX format using XDSCONV. ${ }^{23,24}$

Once one or more processed movies are obtained, a user can either directly solve the data by using the SHELX software suite, or quickly merge multiple pre-processed datasets using XSCALE before submitting to SHELX. By following this screening method, six of the thirty compounds produced $a b$ initio preliminary solutions in an hour or less per sample (Figure 2). The number of movies collected on each of these samples spanned from a single dataset to six datasets. After obtaining these preliminary solutions from SHELXT or SHELXD, the data was refined using SHELXL within ShelXle. ${ }^{24-27}$

A subset of samples subjected to the initial screening at room temperature provided high resolution incident diffraction, but rapid truncation of diffraction resolution was observed over the duration of the continuous rotation movie, presumably due to radiation damage. ${ }^{28}$ (Supporting Information Section 9). Depending on the severity of the beam sensitivity, high quality diffraction data could often still be obtained, but may require extensive data collection and trial-and-error merging of datasets until an optimal combination is achieved. This can be a timeand labor-intensive process. As an alternative strategy, samples can be cooled to cryogenic temperatures to reduce beam sensitivity. Cryogenic screening was avoided except in the case of 

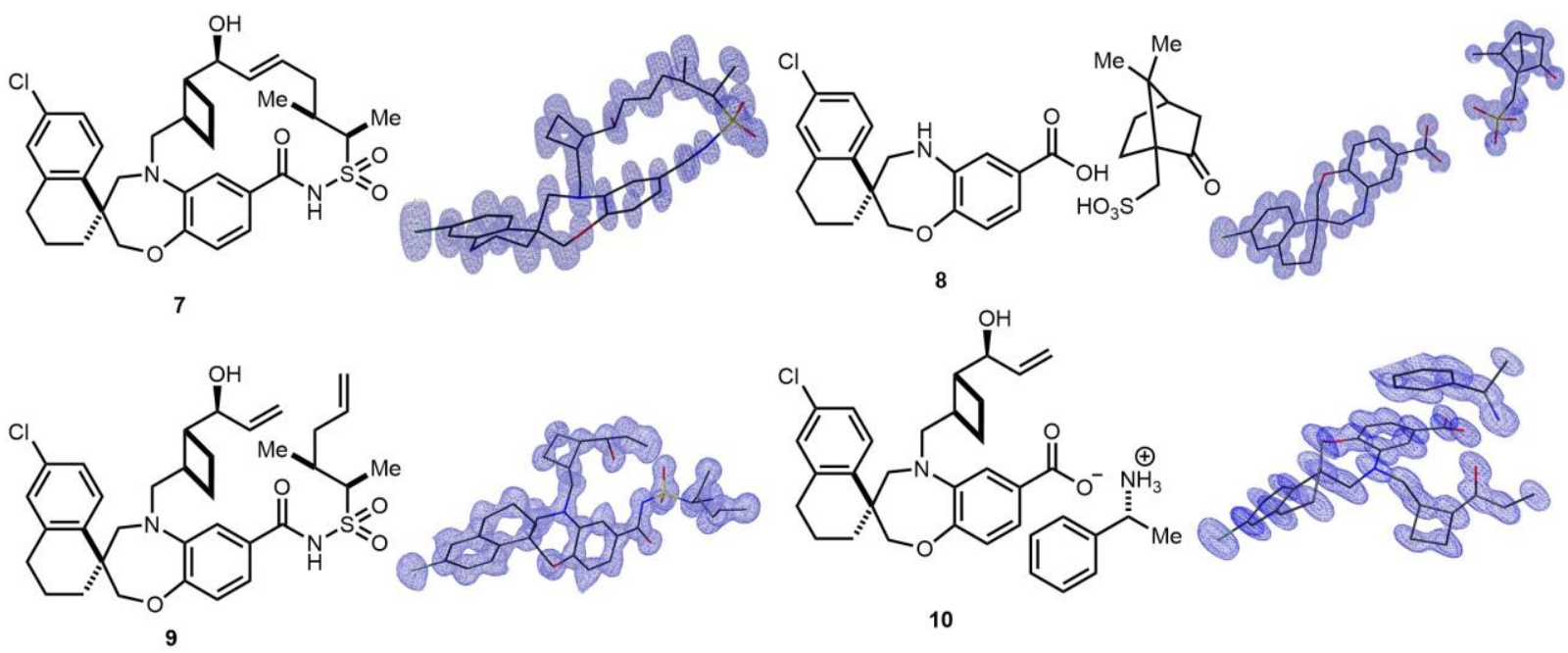

Figure 3. Small molecule structures solved by microED at cryogenic temperatures in under three hours each, with hydrogens omitted for clarity. Crystals of species 7 had two molecules in their asymmetric unit.

these highly beam sensitive samples due to the time required to cool and subsequently warm the cryogenic specimen holder between samples.

Screening at Cryogenic Temperatures. To prepare the next batch of samples, new dry powder grids were prepared as described previously. These grids were placed onto a 626 Gatan cryo holder and inserted into a Talos F200C TEM at room temperature. After insertion but before opening the column valves, the cryo holder was cooled with liquid nitrogen to $-177^{\circ} \mathrm{C}$. The temperature was monitored using a Gatan 1905 Temperature Controller. After stabilizing at cryogenic temperatures, particles were again screened using selected area diffraction. An additional four compounds (7-10, Figure 3) were solved by this method, with a maximum time of three hours from powder to preliminary structure per sample.

Screening Polycrystalline Samples and Recrystallization. The remaining eighteen samples (11-15 Figure 4 and Figure $\mathbf{5 a}-\mathrm{b}$ ) were either too polycrystalline to easily obtain a structure, had poor diffraction resolution, or provided no diffraction at room temperature. In the case of polycrystalline compound 12 (Figure 4), additional hours spent carefully locating monocrystalline domains within a largely polycrystalline sample ultimately provided the structure at room temperature. Other low resolution and polycrystalline samples were subjected to additional screening, but ultimately crystallization attempts were required to provide their solutions.

To crystallize, $\sim 1 \mathrm{mg}$ of powder was placed into $6 \times 50 \mathrm{~mm}$ borosilicate culture tubes. Samples were dissolved in approximately $500 \mathrm{uL}$ of solvent and allowed to slowly evaporate at room temperature. Higher boiling solvents were evaporated from open containers, while low boiling solvents required placing the culture tube inside an empty dram vial with a slightly loosened cap. If the initial solvent failed to produce crystals after fully evaporating, the amorphous samples were re-dissolved in the same culture tube with a new solvent mixture. Evaporation occurred until precipitation was observed. Sample crystallization time spanned from overnight to 3 days. Compound $\mathbf{1 4}$ was crystallized from diethyl ether. The crystals were placed onto a grid as a dry powder, plunge frozen in liquid nitrogen, and transferred into the TEM while the holder was maintained at cryogenic temperatures due to the presumed volatility of the sample. Microcrystals of enantiomeric pair 11 and 13 were obtained from slow evaporation from a 50/50 mixture of $\mathrm{MeCN}$ and $\mathrm{H}_{2} \mathrm{O}$ and dried under reduced pressure. Microcrystals of sample 15 were generated by slow evaporation from a mixture of $\mathrm{H}_{2} \mathrm{O}$ and DMSO (10\% v/v). The crystals were blotted with a kimwipe and dried under reduced pressure to remove excess solvent before being inserted into the TEM.

\section{Results and Discussion.}

Nine datasets were recorded for enantiomeric pair $\mathbf{1}$ and $\mathbf{3}$ (Figure 2), and for each compound two to three of these datasets were merged to provide the structural solutions. Both isomers crystallized in the monoclinic space group $\mathrm{P} 2{ }_{1}$ with similar unit cell parameters. The asymmetric unit of each enantiomer contained two molecules, and overlaying these molecules using PyMol software demonstrated rotational differences that break their crystallographic equivalency. ${ }^{29}$ Enantiomeric pair $\mathbf{2}$ and $\mathbf{4}$ were also obtained during the rapid screening stage, crystallizing in orthorhombic space group $\mathrm{P} 2{ }_{1} 2_{1} 2$ with nearly identical unit cell parameters. Three to four datasets were collected for each enantiomer, but the structure of each enantiomer was resolved using a single dataset. Co-crystallized species 5 was obtained in space group $\mathrm{P} 2_{1}$ after collecting five datasets and merging four for the final solution. This structure assigns connectivity of the spirocyclic compound, but significant rotational disorder in the camphor sulfonic acid (CSA) moiety prevents unambiguous assignment of stereochemistry. Interestingly compound $\mathbf{8}$, which is the enantiomer of compound $\mathbf{5}$, was also studied and required cryogenic data collection due to its beam sensitivity. Compound $\mathbf{6}$ was analyzed after crystallization while its enantiomer, 12, was studied as a powder isolated without crystallization attempts (Figure 4). Only one dataset was collected to generate a single movie solution of crystallized enantiomer 6 (Figure 2) in P $2{ }_{1} 2{ }_{1} 2_{1}$. In contrast, non-crystallized enantiomer 12 (Figure 4) required multiple hours of screening to locate monocrystalline domains. From eight datasets collected, two were merged to provide a solution in space group C2.

Samples that initially looked promising by incident diffraction, but were unable to provide sufficient data at room temperature, had a marked improvement in diffraction 


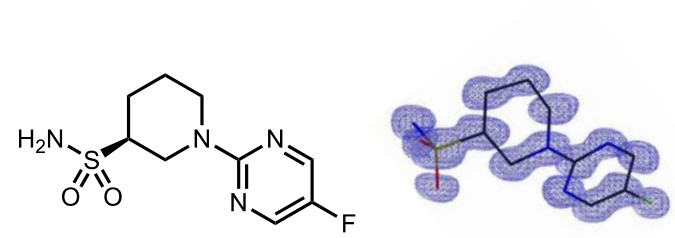

11

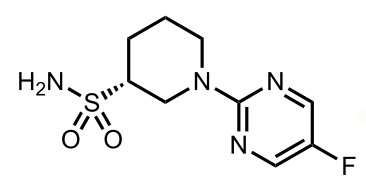

13<smiles>COC(=O)N1CCNCC1CCCCCCO</smiles>

15

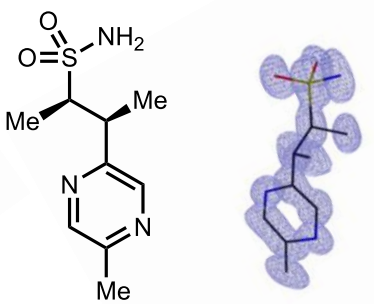

12

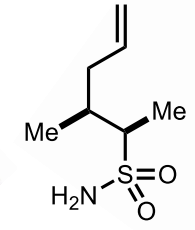

14

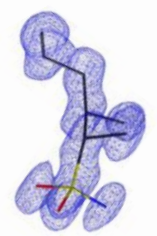

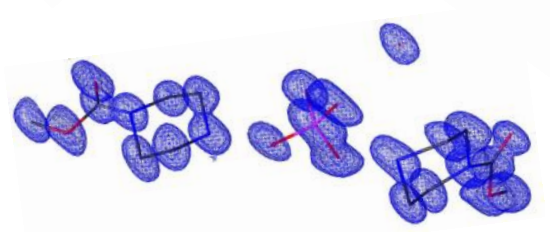

Figure 4. Structures obtained via microED with extensive screening and recrystallization. 12 Obtained by locating monocrystalline species on a largely polycrystalline grid. 11, 13-15 Samples recrystallized to obtain structure. 11 and $\mathbf{1 3}$ contain four molecules within their asymmetric unit. 15 partial view of asymmetric unit. Hydrogens omitted for clarity.

resolution at cryogenic temperatures. Minor variations in the unit cell axes could be observed between room temperature data and cryogenic data, but no phase transitions were observed. Remarkably, the structure of compound 7 (Figure 3 ) containing a 16-membered ring, was able to be solved by SHELXD in $\mathrm{P}_{1}$ after generation of four datasets and merging three. While salt species $\mathbf{8}$ did not provide a solution at room temperature, collection of four datasets (and merging of three) at cryogenic temperatures provided a solution in $\mathrm{P} 2{ }_{1}$ with significantly less rotational disorder at the camphor sulfonic acid molecule than its enantiomeric counterpart, 5 (Figure 2). Diene 9 (Figure 3) yielded a preliminary solution in $\mathrm{P} 2{ }_{1} 2_{1} 2_{1}$ after generating and merging of three datasets. Amine salt 10 required collection of seven and merging of three movies to generate a solution in space group $\mathrm{P} 2{ }_{1} 2{ }_{1}{ }_{1}$. Each of these samples produced a preliminary structure in three hours or less.

To resolve ambiguity of the disordered CSA, 5 was subjected to additional cryogenic screening. Collection of four and merging of two datasets provided a solution with reduced disorder, as is evidenced by the reduction in size of the thermal ellipsoids in the refined structures (See Supporting Information 5.1). It is critical to note that the cryogenic structure of 5 led to unambiguous assignment of the stereochemistry at the CSA, producing the expected enantiomeric structure to 8 ; in contrast, the room temperature solution indicated that chiral salts 5 and $\mathbf{8}$ were diastereomeric structures. Deducing the absolute stereochemistry of the spirocycle from known absolute stereochemistry of the commercial CSA in the room temperature case would have led to misassignment of stereochemistry.
Interestingly, the error values for these two structures are similar, with $\mathrm{R}=0.1198$, GooF $=1.239$ for the room temperature structure, and $\mathrm{R}=0.1127, \mathrm{GooF}=1.143$ for the cryogenic structure.

The primary differences between the two structures of $\mathbf{5}$, other than reduction in size of the thermal ellipsoids, is seen in the preliminary solution obtained with no human input other than molecular formula (See Supporting Information). In the preliminary structures, the cryogenic structure is missing two atoms in the CSA; the room temperature preliminary solution is missing five. Importantly, the missing atoms in the room temperature structure include those relevant to assigning stereochemistry.

While the spirocycle connectivity is well-resolved in both structures, we believe this serves as an excellent example as to why it is critical for microED reports to not only include standard crystallographic statistics, but preliminary structural solutions and refined structure files as well.

While eleven structures were obtained directly from the samples as received, structural elucidation of four additional compounds was achieved through crystallization screening. For example, sulfonamide pair $\mathbf{1 1}$ and $\mathbf{1 3}$ (Figure 4) were recrystallized from $\mathrm{MeCN} / \mathrm{H}_{2} \mathrm{O}$ and provided solutions in $\mathrm{P} 2{ }_{1} 2{ }_{1} 2$. While screening recrystallized $\mathbf{1 3}$, multiple similar unit cells were encountered, suggesting polymorphism. The structure of the presumed polymorph was not able to be determined. This polymorphism was not detected in the original sample. Structure $\mathbf{1 5}$ is another example of a process sample that provided structural information but was not representative 
a)

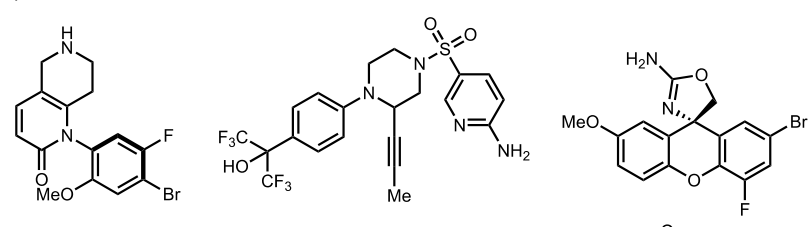

b)

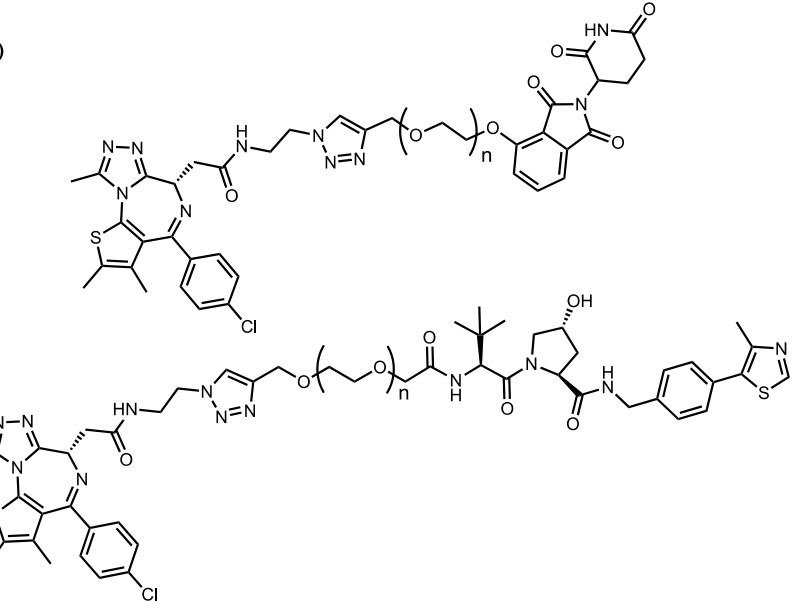

Figure 5. Samples that failed to generate structures. a Enantiomeric pairs (only one enantiomer drawn) $\mathbf{b}$ PROTAC, $n=0-4$.

of the original sample. While the presence of piperazine is maintained at levels lower than 5000 ppm, this impurity was found in significant amounts in the structure obtained from recrystallization. This highlights a limitation in gaining information about the bulk sample from recrystallized samples analyzed by microED.

The remaining fifteen compounds failed to produce structures in our hands in a timely manner. These samples are comprised of three small molecule enantiomeric pairs (Figure 5a) and nine PROTAC compounds (Figure 5b). While most medicinal and process small molecule samples can be evaluated as dry powders, complex species like PROTACs are assumed to benefit from evaluation in the frozen hydrated state. Extensive crystallization and vitrification screening is a potential avenue for producing microcrystals from these highly complex species, but was beyond the scope of this rapid-timeline study where fifteen of thirty compounds were solved using approximately forty hours of TEM time and seventy hours of automated and userdriven data processing.

\section{Conclusions.}

This study highlighted the utility of using microED as a tool for rapid structural analysis and elucidation of pharmaceutical compounds representing a range of chemical complexity. While study of biological samples via microED often requires timeconsuming vitrification and cryo transfer processes, many industrial compounds explored in this study provided diffraction data sufficient for obtaining preliminary solutions within minutes to a few hours. Interestingly, all samples procured from process development provided solutions, albeit two of them requiring additional crystallization. From thirty samples analyzed, the structures of fifteen were determined in this study. Of the small molecule subset, this translated to a success rate of approximately $70 \%$. The majority of the structures were obtained from microcrystalline powders either not subjected to any crystallization, or from compounds crystallized for the purpose of purification. The speed and minimal sample preparation required for this process demonstrates that microED has significant promise as a routine analytical tool for unambiguous structural elucidation in the pharmaceutical pipeline.

\section{ASSOCIATED CONTENT}

\section{Supporting Information}

The Supporting Information is available free of charge on the ACS Publications website.

Experimental details, transmission electron microscope images and diffraction data, automated data processing source code, and crystallographic data (PDF)

\section{AUTHOR INFORMATION}

\section{Corresponding Author}

*hosea@chem.ucla.edu

*quasdorf@amgen.com

*jess@microedlab.com

\section{Present Addresses}

$\dagger$ Current address for S.D.W is

Johnson Matthey

25 Patton Road

Devens, MA 01434

Shawn.Walker@matthey.com

$\dagger$ Current address for V.J.C. is

Oncovalent Therapeutics

1290 Rancho Conejo Blvd

Thousand Oaks, CA 91320

vcee@oncovalent.com

$\dagger$ Current address for A.G.S is

Loxo Oncology at Lilly

Process Chemistry CMC

Boulder, CO 80301

Asmith@loxooncology.com

†Current address for H.M.N. is hosea@ caltech.edu 


\section{Author Contributions}

H.M.N. and K.Q. conceived the idea and supervised the project. J.E.B. performed the described experiments and analyzed the data. The manuscript was written through contributions of all authors.

\section{Notes}

J.E.B., H.M.N., J.R., and D.G. are founders of MicroEDLab.com, a for-profit contract research organization providing electron microscopy structural solutions.

\section{ACKNOWLEDGMENT}

Financial support for this work was generously provided by the David and Lucile Packard Foundation (to H.M.N. \& J.R.), the Arnold and Mabel Beckman Foundation (to J.R.) and the National Science Foundation (DGE-1650604 to J.E.B.)

\section{REFERENCES}

(1) Lee, A. Y.; Erdemir, D.; Myerson, A. S. Crystal polymorphism in chemical process development. Annu. Rev. Chem. Biomol. Eng. 2011, 2, 259-280.

(2) Caille, S.; Cui, S.; Faul, M. M.; Mennen, S. M.; Tedrow, J. S.; Walker, S. D. Molecular complexity as a driver for chemical process innovation in the pharmaceutical industry. J. Org. Chem. 2019, 84, $4583-4603$

(3) Dunitz, J. D. X-ray Analysis and the Structure of Organic Molecules; Verlag: Zürich, 1995.

(4) Florence, A.; Johnston, A.; Fernandes, P.; Shankland, K.; Stevens, H. N. E.; Osmundsen, S.; Mullen, A. B. Powder study of hydrochlorothiazide form II. Acta Cryst. E 2005, 61, 2798-2800.

(5) Harris, K. D. M. Structure solution from powder X-ray diffraction data by genetic algorithm techniques, applied to organic materials generated as polycrystalline products from solid state processes. Mat. Man. Proc. 2009, 24, 293-302.

(6) Miclaus, M.; Grosu, I. G.; Filip, X.; Tripon, C.; Filip, C. Optimizing structure determination from powders of crystalline organic solids with high molecular flexibility: the case of lisinopril dehydrate. CrystEng Comm. 2014, 16, 299-303.

(7) Luedeker, D.; Gossmann, R.; Langer, K.; Brunklaus, G. Crystal Engineering of Pharmaceutical Co-crystals: "NMR Crystallography" of Niclosamide Co-crystals. Cryst. Growth Des. 2016, 16, 3087-3100.

(8) Hope, M. A.; Nakamura, T.; Ahlawat, P.; Mishra, A.; Cordova, M.; Jahanbakhshi, F.; Mladenović, M.; Runjhun, R.; Merten, L.; Hinderhofer, A.; Carlsen, B. I.; Kubicki, D. J.; Gershoni-Poranne, R.; Schneeberger, T.; Carbone, L. C.; Liu, Y.; Zakeeruddin, S. M.; Lewinski, J.; Hagfeldt, A.; Schreiber, F.; Rothlisberger, U.; Grätzel, M.; Milić, J. V.; Emsley, L. Nanoscale phase segregation in supramolecular p-templating for hyrbide perovskite photovoltaics from NMR crystallography J. Am. Chem. Soc. 2021, 143, 1529-1538.

(9) David, W. I. F.; Shankland, K. Structure determination from powder diffraction data. Acta Cryst. A 2008, 64, 52-64.

(10) Jones, C. G.; Martynowycz, M. W.; Hattne, J.; Fulton, T. J.; Stoltz, B. M.; Rodriguez, J. A.; Nelson, H. M.; Gonen, T. The CryoEM Method MicroED as a Powerful Tool for Small Molecule Structure Determination. ACS Cent. Sci. 2018, 4, 1587-1592.
(11) Gemmi, M.; Mugnaioli, E.; Gorelik, T. E.; Kolb, U.; Palatinus, L.; Boullay, P.; Hovmöller, S.; Abrahams, J. P. 3D Electron Diffraction: The Nanocrystallography Revolution. ACS Cent. Sci. 2019, 5 , $1315-1329$

(12) Mugnaioli, E.; Lanza, A. E.; Bortolozzi, G.; Righi, L.; Merlini, M.; Cappello, V.; Marini, L.; Athanassiou, A.; Gemmi, M. Electron Diffraction on Flash-Frozen Cowlesite Reveals the Structure of the First Two-Dimensional Natural Zeolite. ACS Cent. Sci. 2020, 6, 15781586.

(13) Clabbers, M. T. B.; Hongyi, X. Microcrystal electron diffraction in macromolecular and pharmaceutical structure determination. Drug Discovery Today: Technologies. 2020, In Press.

(14) Curtis, B. J.; Kim, L. J.; Wrobel, C. J. J.; Eagen, J. M.; Smith, R. A.; Burch, J. E.; Le, H. H.; Artyukhin, A. B.; Nelson, H. M.; Schroeder, F. C. Identification of Uric Acid Gluconucleoside-Ascaroside Conjugates in Caenorhabditis elegans by Combining Synthesis and MicroED Org. Lett. 2020, 22, 6724-6728.

(15) Jones, C. G.; Asay, M.; Kim, L. J.; Kleinsasser, J. F.; Saha, A.; Fulton, T. J.; Berkley, K. R.; Cascio, D.; Malyutin, A. G.; Conley, M. P.; Stoltz, B. M.; Lavallo, V.; Rodríguez, J. A.; Nelson, H. M. Characterization of Reactive Organometallic Species via MicroED. ACS Centr. Sci. 2019, 5, 1507-1513.

(16) Das, P. P.; Mugnaioli, E.; Nicolopoulos, S.; Tossi, C.; Gemmi, M.; Galanis, A.; Borodi, G.; Pop, M. M. Crystal Structures of Two Important Pharmaceuticals Solved by 3D Precession Electron Diffraction Tomography. Org. Proc. Res. \& Dev. 2018, 22, 1365-1372.

(17) Das, P. P.; Perez, A. G.; Galanis, A. S.; Nicolopoulos, S. Structural Characterization of Beam Sensitive Pharmaceutical Compounds Using 3D Electron Diffraction-Micro-ED at Low Dose with Pixelated Detectors. Microscopy and Microanalysis. 2020, 26, 1522-1522.

(18) Brázda, P.; Palatinus, L.; Babor, M. Electron diffraction determines molecular absolute configuration in a pharmaceutical nanocrystal. Science. 2019, 364, 667-669.

(19) Kim, L. J.; Ohashi, M.; Zhang, Z.; Tan, D.; Asay, M.; Cascio, D.; Rodriguez, J. A.; Tang, Y.; Nelson, H. M. Prospecting for natural products by genome mining and microcrystal electron diffraction. Nat. Chem. Biol. 2021, 17, 872-877.

(20) Wang, B.; Zou, X.; Smeets, S. Automated serial rotation electron diffraction combined with cluster analysis: an efficient multi-crystal workflow for structure determination IUCrJ. 2019, 6, 854-867.

(21) Hattne, J.; Reyes, F. E.; Nannenga, B. L.; Shi, D.; de la Cruz, M. J.; Leslie, A. G. W.; Gonen, T. MicroED data collection and processing. Acta Cryst. A 2015, 71, 353-360.

(22) Kabsch, W. Xds Acta Cryst. D 2010, 66, 125-132.

(23) Kabsch, W. Integration, scaling, space-group assignment and post-refinement Acta Cryst. D 2010, 66, 133-144.

(24) Sheldrick, G. M. A short history of SHELX. Acta Cryst. A 2008, $64,112-122$.

(25) Sheldrick, G. M. SHELXT - Integrated space-group and crystal-structure determination. Acta Cryst A. 2015, 71, 3-8.

(26) Sheldrick, G. M. Crystal structure refinement with SHELXL. Acta Cryst. C 2015, 71, 3-8.

(27) Hübschle, C. B., Sheldrick, G. M. \& Dittrich, B. ShelXle: A Qt graphical user interface for SHELXL. J. Appl. Cryst. 2011, 44, 12811284.

(28) Hattne, J., Shi, D., Glynn, C., Zee, C.-T., Gallagher-Jones, M., Martynowycz, M. W., Rodriguez, J. A., Gonen, T. Analysis of Global and Site-Specific Radiation Damage in Cryo-EM Structure 2018, 5, 759-766.

(29) Delano, W. The PyMOL Molecular Graphics System (Schrodinger LLC). http://www.pymol.org. 